\title{
Ablation Outcomes for Atypical Atrial Flutter versus Recurrent Atrial Fibrillation Following Index Pulmonary Vein Isolation
}

\author{
Tauseef Akhtar ${ }^{1}$, Usama Daimee ${ }^{2}$, Bhradeev Sivasambu ${ }^{3}$, Thomas Boyle ${ }^{2}$, Armin \\ Arbab-Zadeh ${ }^{2}$, Joseph Marine ${ }^{4}$, Ronald Berger ${ }^{4}$, Hugh Calkins ${ }^{4}$, and David Spragg ${ }^{4}$ \\ ${ }^{1}$ Johns Hopkins School of Medicine \\ ${ }^{2}$ Johns Hopkins University School of Medicine \\ ${ }^{3}$ Johns Hopkins Medicine \\ ${ }^{4}$ Johns Hopkins Hospital
}

December 22, 2020

\begin{abstract}
Background: Data related to electrophysiologic characteristics of atypical atrial flutter (AFL) following atrial fibrillation (AF) ablation and its prognostic value on repeat ablation success are limited. Methods: We studied consecutive patients undergoing a repeat LA ablation for either recurrent AF or atypical AFL, following 3 months after index AF ablation, between January 2012 and July 2019. The demographics, procedural data, complications, and 1-year arrhythmia-free survival rates were recorded for each subject after the first repeat ablation. Results: Of the total 336 included patients, 102 underwent a repeat ablation for atypical AFL and 234 for recurrent AF. The mean age was 63.7 ? 10.7 years, and $72.6 \%$ of patients were male. The atypical AFL cohort had significantly higher LA diameters (4.6 vs. $4.4 \mathrm{~cm}, \mathrm{p}=0.04)$ and LA volume indices $(\mathrm{LAVi} ; 85.1 \mathrm{vs} .75 .4 \mathrm{ml} / \mathrm{m} 2$, $\mathrm{p}=0.03$ ) compared to AF patients at repeat ablation. Atypical AFLs were roof-dependent in 35.6\% and peri-mitral in $23.8 \%$ of cases. Major complications at repeat ablation occurred in $0.9 \%$ of the total cohort. Arrhythmia-free survival at one year was significantly higher in the recurrent atypical AFL than the recurrent AF cohort (75.5 vs. 65.0 \%, p=0.04). Conclusion: In our series, roof-dependent flutter is the most common form of atypical atrial flutter post AF ablation. Patients developing atypical AFL after index AF ablation have greater LA dimensions than patients with recurrent AF. The success rate of first repeat ablation is significantly higher among patients with recurrent atypical AFL compared to recurrent AF after index AF ablation.
\end{abstract}

Title: Ablation Outcomes for Atypical Atrial Flutter versus Recurrent Atrial Fibrillation Following Index Pulmonary Vein Isolation

Short Title: Impact of recurrent atypical atrial flutter on repeat ablation

Tauseef Akhtar MD, Usama A. Daimee MD, Bhradeev Sivasambhu MD, Thomas A. Boyle MD, Armin Arbab-Zadeh MD, MPH, PhD, Joseph E. Marine MD, Ronald Berger MD, PhD, Hugh Calkins MD, David Spragg MD

Division of Cardiology, Johns Hopkins University School of Medicine, Baltimore, MD, 21287 USA.

\section{Authors have no conflicts to disclose.}

Funding for this research was provided in part by the Edward St. John Fund for AF Research, the Roz and Marvin H. Weiner and Family Foundation, the Dr. Francis P. Chiaramonte Foundation, the Marilyn and Christian Poindexter Arrhythmia Research Fund, Norbert and Louise Grunwald Cardiac Arrhythmia Research Fund, and the Mr. \& Mrs. Larry Small AF Research Fund. Dr. Arbab-Zadeh discloses research 
grant support from Canon Medical Systems. All other authors have reported that they have no relationships relevant to the contents of this paper to disclose.

\title{
Word count: 3538
}

\section{For correspondence:}

Tauseef Akhtar MD

Johns Hopkins Hospital

600 N. Wolfe St., Baltimore MD 21287

Phone: 2814646952

tausif.akhtar@gmail.com

\begin{abstract}
Background: Data related to electrophysiologic characteristics of atypical atrial flutter (AFL) following atrial fibrillation $(\mathrm{AF})$ ablation and its prognostic value on repeat ablation success are limited.
\end{abstract}

Methods: We studied consecutive patients who underwent a repeat left atrial (LA) ablation procedure for either recurrent AF or atypical AFL, at least 3 months after index AF ablation, between January 2012 and July 2019. The demographics, clinical history, procedural data, complications, and 1-year arrhythmia-free survival rates were recorded for each subject after the first repeat ablation.

Results: A total of 336 patients were included in our study. Among these 336 patients, 102 underwent a repeat ablation procedure for atypical AFL and 234 underwent a repeat ablation procedure for recurrent AF. The mean age was $63.7 \pm 10.7$ years, and $72.6 \%$ of patients were male. The atypical AFL cohort had significantly higher LA diameters (4.6 vs. $4.4 \mathrm{~cm}, \mathrm{p}=0.04$ ) and LA volume indices (LAVi; 85.1 vs. 75.4 $\mathrm{ml} / \mathrm{m}^{2}, \mathrm{p}=0.03$ ) compared to AF patients at repeat ablation. Atypical AFL patients were more likely to have had index radiofrequency (RF) ablation (as opposed to cryoballoon) than recurrent AF patients (98\% vs. $81 \%, \mathrm{p}=0.01$ ). Atypical AFLs were roof-dependent in $35.6 \%$ and peri-mitral in $23.8 \%$ of cases. Major complication at repeat ablation occurred in $0.9 \%$ of the total cohort. Arrhythmia-free survival at one year was significantly higher in the recurrent atypical AFL compared to the recurrent AF cohort (75.5 vs. 65.0 $\%, \mathrm{p}=0.04)$.

Conclusion: In our series, roof-dependent flutter is the most common form of atypical atrial flutter post AF ablation. Patients developing atypical AFL after index AF ablation have greater LA dimensions than patients with recurrent AF. The success rate of first repeat ablation is significantly higher among patients with recurrent atypical AFL as compared to recurrent AF after index AF ablation.

\section{Keywords:}

Atrial fibrillation, Atypical atrial flutter, Roof dependent atrial flutter, Perimitral atrial flutter, Left atrial volume index, Radiofrequency ablation

\section{Abbreviations and Acronyms}

$$
\begin{aligned}
& \mathrm{AF}=\text { atrial fibrillation } \\
& \mathrm{AFL}=\text { atrial flutter } \\
& \mathrm{LA}=\text { left atrium } \\
& \mathrm{LAVi}=\text { left atrial volume index } \\
& \mathrm{RF}=\text { radiofrequency } \\
& \mathrm{CB}=\text { cryoballoon }
\end{aligned}
$$


TEE $=$ trans esophageal echocardiogram

$\mathrm{TTE}=$ trans thoracic echocardiogram

$\mathrm{PVI}=$ pulmonary vein isolation

$\mathrm{PAF}=$ paroxysmal atrial fibrillation

PsAF $=$ persistent atrial fibrillation

\section{Introduction}

Catheter ablation (CA) of atrial fibrillation (AF) is a well-established procedure for the treatment of drug refractory AF. Single-procedure AF ablation is successful in 60-80\% of optimal candidates, but many patients require repeat ablation(1). Atypical atrial flutter (AFL) has been reported in approximately $8 \%$ of patients following AF ablation using RF energy(2). Patients with AFL can be very symptomatic and refractory to medical therapy, often requiring repeat ablation procedures. While it is a common belief that the outcomes for repeat ablation procedures in patients who present with atypical AFl are superior to those observed in patients who present with recurrent AF, there are limited data available to support this assumption. This study aimed to describe the electrophysiological findings and the prognostic utility of recurrent atypical AFL versus recurrent $\mathrm{AF}$ after index $\mathrm{AF}$ ablation in a large contemporary cohort of patients at our center.

\section{Methods}

\section{Study Cohort}

We conducted a single-center, retrospective cohort study comprising patients, derived from an IRB-approved, prospectively populated clinical database of AF ablation patients. All patients underwent first repeat AF or atypical AFL ablation between January 2012 to July 2019 at the Johns Hopkins Hospital. Of the 336 patients who participated in this analysis, 289 had an index PVI with RF energy, while 46 had index cryoballoon ablation. The total patient cohort was divided into two groups (recurrent AF and atypical AFL) based on the recurrent arrhythmia identified during clinical follow-up and prompting repeat ablation. Patients presenting with episodes of both AF and atypical AFL were included in the AF cohort.

The demographics, clinical history, procedural data, complications, and outcomes were recorded for each case. Patients found to have typical AFL only at repeat ablation were excluded from this analysis. Arrhythmia recurrence and peri-procedural complications were ascertained based on monitoring strategies put forth in the 2017 Heart Rhythm Society(1) (HRS) consensus document. Arrhythmia recurrence was defined as any AF or atrial tachyarrhythmia (AT) sustained for $>30$ s recorded by a surface electrocardiogram or rhythm monitoring device after a 90-day blanking period. Procedure-related complications, such as major bleeding, minor bleeding, phrenic nerve palsy, cerebral embolism, pericardial effusion/tamponade, atrioesophageal fistula, or extended hospitalization, were assessed.

\section{Left Atrial Size Assessment}

LA diameter was assessed with a pre-procedural transthoracic echocardiography (TTE; $\mathrm{n}=256$ ), when available. LA diameter $>4 \mathrm{~cm}$ on TTE was defined as enlarged based on the American Society of Echocardiography guidelines (3). LA volume was measured using pre-procedural contrast-enhanced cardiac computed tomographic $(\mathrm{CT})$ imaging $(\mathrm{n}=236)$, when available. CT images were obtained using a 320-detector scanner (Aquilion ONE, Canon Medical Systems) and prospective scan acquisition targeted at $40 \%$ of the R-R cycle (end-systole) without padding. Images were reconstructed with a slice thickness of $1 \mathrm{~mm}$ and soft reconstruction kernel (FC03) and then transferred to a commercially available image workstation (Vitrea, Vital Images, Minneapolis, MN, USA) for analysis. The software includes a cardiac chamber volume analysis algorithm, which allows automated tracing of the contrast enhanced left atrial cross-sectional border contours and computes volume by interpolating the vertical increments of $1 \mathrm{~mm}$ for the structure's cranio-caudal extension. The algorithm truncates borders in the proximal portion of the pulmonary veins and includes the 
left atrial appendage for volumetric assessment. Indexed volumes were calculated by dividing the volumes by body surface area and recorded as LA volume index (LAVi).

\section{Repeat Mapping and Ablation Strategy}

All repeat ablations for patients in this study were performed using RF energy. Femoral site access was obtained, and intravenous heparin was administered to maintain activated clotting times $>350 \mathrm{~s}$. After performing a double transseptal puncture, a Lasso or PentaRay mapping catheter (Biosense Webster, Diamond Bar, California) was positioned in the left atrium. An electroanatomic map of the left atrium was obtained using the CARTO system (Biosense Webster) and superimposed on pre-acquired cardiac CT image.

AFL Cohort : In all AFL patients, simultaneous voltage and activation mapping was performed in patients presenting in AFL. Patients presenting in sinus rhythm underwent a flutter induction protocol consisting of both atrial burst pacing and atrial programmed stimulation until flutter was successfully initiated. Activation mapping of AFL was supplemented with entrainment mapping from putative critical isthmuses to further categorize flutter circuits. Based on the location of critical isthmus identification, flutters were classified as 1) peri-mitral, 2) roof-dependent, and 3) other (including multi-loop circuits, idiosyncratic flutters involving ablation lesion sets, and transitioning flutters with more than one stable circuit). All flutter forms occurring spontaneously or resulting from radiofrequency (RF) delivery during the first repeat ablation were documented. Following conclusion of targeted AFL ablation, PV isolation was assessed and addressed as needed (below).

AFL ablation was performed targeting the critical isthmus with a transecting lesion anchored to either preexisting anatomical structures (i.e., mitral valve annulus) or with iatrogenic scars (e.g., left PV ostia after pulmonary vein isolation (PVI)), generating linear ablation lines. Conduction block across the ablation line was verified by $3 \mathrm{D}$ electroanatomical mapping with a multipolar mapping catheter in normal sinus rhythm (NSR). RF ablation was performed until (a) the tachycardia terminated or (b) converted into a second form, identified by a significant change in CS activation or in combination with alteration of surface electrocardiogram (ECG) morphology. Termination of the tachycardia was considered as ablation success if it occurred directly during RF delivery with or without prior prolongation of tachycardia cycle length (TCL) and in the absence of a premature atrial beat ([?]90\% TCL). Reinduction protocols were performed at the discretion of the operator.

AF Cohort : PVs were assessed for reconnection in all patients, and re-isolation was performed for those showing the electrical reconnection. A 4-mm, open-irrigated, contact force-sensing RF catheter (ThermoCool SmartTouch, Biosense Webster) was used, and re-isolation of the PVs was performed using a real-time automated display of RF application points (Visitag, Biosense Webster) with predefined catheter stability settings. Starting energy delivery parameters were 25 to 35 watts on the posterior LA wall and 35 to 45 watts at other sites. The target contact force was between 5 and $20 \mathrm{~g}$ at all sites. Esophageal temperature was monitored, and the RF delivery paused if the esophageal temperature increased by $0.5 \mathrm{deg}$ C. In patients with recurrent $\mathrm{AF}$, but with durable isolation of the PVs, additional substrate modification was performed in accordance with consensus guidelines. ${ }^{1}$ Additional ablation consisted of lesions involving the roof, floor, posterior wall, mitral isthmus, cavotricuspid isthmus (CTI), superior vena cava (SVC), coronary sinus (CS), and others, including the complex fractionated atrial electrograms (CFAE), were performed at the discretion of the operator. Acute procedural success was defined as electrical isolation of PVs, confirmed by entrance block to individual PVs, and a bidirectional block line when linear ablations were performed.

In the AF cohort, repeat ablation strategies were classified into 1) PVI only, 2) PVI plus additional ablation, and 3) additional ablation only.

\section{Clinical Follow-up}

All patients were observed in the hospital for a minimum of one-night post-ablation. Routine

follow-up (history, exam, and electrocardiography or Holter) was performed to detect clinically 
relevant recurrence of arrhythmia, in a manner consistent with the AF Expert Consensus Statement (1). All patients were seen at the outpatient clinic or by a local cardiologist at 3, 6, and 12 months, and additionally, if prompted by symptoms. Event monitors were arranged for patients in whom symptoms suggestive of recurrence developed in the post-blanking phase of follow-up. If present at the time of ablation, antiarrhythmic drug therapy was discontinued at the 3-month follow-up visit. One-year outcomes were assessed in all patients via clinic follow-up, electronic health record review, or phone interview.

\section{Statistical Analysis}

Continuous variables were described with measures of central tendency and dispersion (mean and standard deviation). Categorical variables were described as frequencies or mode when appropriate. Continuous variables were evaluated by $t$-test, and categorical variables compared with $\chi^{2}$ or Fisher's exact test. The cumulative probability of survival free from atrial arrhythmia was displayed according to the Kaplan-Meier method, with comparisons of cumulative event rates by the log-rank test. Follow-up for all patients was censored at one year after repeat ablation. A p-value of $<0.05$ was considered statistically significant. All analyses were performed using SPSS Statistics Software for Windows version 23.0 (IBM Corporation, Armonk, New York).

\section{Results}

\section{Baseline Clinical Characteristics}

A total of 336 patients underwent first repeat ablation for recurrent AF or atypical AFL at our center from January 2012 to July 2019. Of these, 234 (69.6\%) patients underwent repeat ablation for AF, and 102 $(30.3 \%)$ underwent repeat ablation for atypical AFL. The mean age was $63.7 \pm 10.7$ years in the total cohort, and the majority were white and male (Table 1). The mean body mass index (BMI) was $29.9 \pm 5.5 \mathrm{~kg} / \mathrm{m}^{2}$, and mean CHA2DS2-VASc score was $2 \pm 1.4$, respectively, with no significant difference between cohorts. Patients with atypical AFL were older than those with recurrent AF (65.6 vs. 62.9 years, $p=0.03$ ) and had a greater proportion of diabetics (22.1 vs. $11.0 \%, \mathrm{p}=0.01)$. A greater proportion of the patients in the recurrent atypical AFL cohort had a history of prior cardioversion ( 58.8 vs. $43.2 \%, \mathrm{p}=0.01$ ) and cardioversions in the preceding one year (47.1 vs. $28.2 \%, \mathrm{p}<0.01)$ respectively. Similarly, a greater proportion of the patients in the recurrent atypical AFL cohort were not prescribed antiarrhythmic drugs (AAD) before the repeat ablation (43.1 vs. $29.0 \%, \mathrm{p}<0.01$ ) as compared to the recurrent $\mathrm{AF}$ cohort. Other comorbidities remained comparable between the two cohorts.

\section{Imaging Characteristics}

The mean LA diameter for the total cohort was $4.4 \pm 0.7 \mathrm{~cm}$ (Table 2). The mean LAVi was $77.5 \pm 26.2$ $\mathrm{ml} / \mathrm{m}^{2}$. The mean left ventricular ejection fraction (LVEF) was $57.2 \pm 9.1 \%$. Patients presenting with atypical AFL had greater mean LA diameter (4.6 vs. $4.4 \mathrm{~cm}, \mathrm{p}=0.04)$ and LAVi ( 85.1 vs. $75.4 \mathrm{ml} / \mathrm{m}^{2}$, $\mathrm{p}=0.03)$ compared to the recurrent AF cohort. Patients in the recurrent atypical AFL cohort had decreased LVEF (55.1 vs. $58.2 \%, \mathrm{p}=0.01$ ) compared to the recurrent AF cohort.

\section{Characteristics of Recurrent Atypical AFL}

Among the 102 patients who underwent repeat ablation for atypical AFL, 79 patients presented in AFL on the day of repeat ablation, and 23 presented in sinus rhythm (Figure 1). For those patients presenting in NSR, AFL was successfully induced with standard pacing protocols in 14/23 patients. Most patients had at least one documented atypical AFL, and the number of flutter circuits ranged from 0 to 5 (Table 3). Atypical AFL could not be documented in $9(8.7 \%)$ of the patients during the ablation. A total of $36(35.6 \%)$ of the patients had roof dependent flutters. Peri mitral flutters were seen in $24(23.8 \%)$ of the patients, and 33 
(32.7\%) patients had other flutter forms (idiosyncratic flutter circuits involving prior lesion sets; multipleloop flutter circuits; transitioning flutters that were not durable enough for adequate mapping).

\section{Initial Ablation Characteristics and Interval Between Initial and Repeat Ablation}

Among the 336 patients in this study, 213 underwent an initial AF ablation for paroxysmal AF (PAF), and 123 had an initial AF ablation for persistent AF (PsAF) (Table 4). The proportion of patients with PAF and PsAF at the time of index ablation was comparable among the patients undergoing repeat ablation for AF and atypical AFL. The index ablation procedure was performed with RF energy in 190 (81.2\%) of 234 of the patients undergoing repeat ablation for AF and 99 (98\%) of 102 patients undergoing repeat ablation for atypical atrial flutter $(\mathrm{p}<0.01)$. The remainder of the patients in both groups had an initial AF ablation with the cryoballoon system. For the total cohort, the mean duration between index and repeat ablation was $2.1 \pm 2.9$ years. There was no difference in the interval between the index and repeat ablation in recurrent AF vs. atypical AFL cohorts (2.2 vs. 1.9 years, $\mathrm{p}=0.29$ ).

\section{Ablation Procedure and Acute Outcomes}

Among the 102 patients in the recurrent atypical AFL cohort, acute ablative success was achieved in 74 $(73.3 \%)$ (Table 3). Five (5\%) patients had rhythm conversion to AF during flutter ablation, and $5(5 \%)$ patients had spontaneous termination of atypical AFL. A total of $9(8.9 \%)$ patients required cardioversion to achieve NSR. Acute procedural success was achieved in $100 \%$ of patients in the recurrent AF cohort (Table 4). Of the 234 patients undergoing repeat ablation for recurrent AF, PV reconnection was seen in $196(83.7 \%)$. Four, 3, 2, and 1 reconnected PVs were seen in $42.3 \%, 12 \%, 21.7 \%$, and $7.7 \%$ of recurrent AF patients, respectively. In the 102 AFL patients, recurrent connection of one or more PVs was seen in $76.4 \%$. The mean number of PVs showing electrical reconnection was significantly higher among patients in the recurrent AF cohort (2.8 vs. $2.3, \mathrm{p}<0.01)$ than the recurrent atypical AFL cohort. Similarly, the proportions of all the PVs with electrical reconnection were also higher in the AF cohort.

As anticipated, a greater proportion of patients in the recurrent atypical AFL cohort required PVI plus additional ablation $(70.2$ vs. $50.4 \%, \mathrm{p}=0.00)$ and additional ablation strategies $(12.7$ vs. $0.4 \%, \mathrm{p}<0.01)$ compared to the recurrent AF cohort. The use of mitral isthmus line ablation (13.4 vs. $0.4 \% \%, \mathrm{p}<0.01$ ), roof ablation ( 47.1 vs. $26.5, \mathrm{p}<0.01$ ) and combination of different non-PV ablations ( 46.1 vs. $19.7 \%, \mathrm{p}=0.00$ ) were significantly higher among patients in the recurrent AFL cohort.

There were three $(0.9 \%)$ patients with major complications in the total cohort undergoing redo ablation, and the incidence of complications remained comparable between the recurrent AF and AFL cohort. One patient suffered transient right phrenic nerve paralysis that resolved after two months, and two patients developed access site pseudoaneurysms treated successfully with thrombin injections. There were no instances of cardiac tamponade or perforation, PV stenosis, stroke/TIA, atrio-esophageal fistula, or procedure-related deaths.

\section{Efficacy of Ablation with Follow-up}

Freedom from atrial arrhythmia at one year of follow-up in the total cohort was $68.2 \%$. Patients undergoing repeat ablation for atypical AFL had significantly higher arrhythmia free survival rate (75.5 vs. $65.0 \%$, $\mathrm{p}=0.04$ ) as compared to patients undergoing repeat ablation for AF (Figure 2) at one year of follow-up.

\section{Discussion}

Whether patients returning after index PVI with new atypical AFL represent an attractive target for redo ablation has not been the subject of rigorous investigation. We sought to investigate the rates of arrhythmiafree 1y survival in two cohorts of patients presenting with recurrent arrhythmia following PVI: those with atypical AFL only, and those with recurrent AF (with or without flutter). The main findings of our study are: 1) Patients undergoing repeat ablation for atypical AFL after index AF ablation have enlarged LA and higher LAVi; 2) Use of RF energy in the index AF ablation is higher among patients developing recurrent atypical AFL; 3) Isolated roof dependent AFL and peri-mitral AFL account for roughly $60 \%$ for post-PVI 
flutters observed; 4) 1-year arrhythmia free survival rate is higher among patients undergoing repeat ablation for atypical AFL as compared to recurrent AF.

We found that LA diameter and LA volume was significantly greater among patients developing atypical AFL as compared to AF after the index ablation. This finding is in line with previous studies which reported that greater LA diameter(4) and LAVi(5) independently predict de novo atypical AFL, highlighting the role of intrinsic structural alteration in mediating fixed reentry.

We also observed that the use of RF energy for index ablation was significantly higher among the patients developing recurrent atypical AFL as compared to recurrent AF. A number of previous studies have reported the factors predictive of atypical AFL recurrence after AF ablation(6-8), and few of the studies(9,10) evaluated the predictive value of energy source (RF vs. cryo) during AF ablation. Julia et al (9); reported in their study that RF AF ablation is associated with a higher incidence of recurrent atypical AFL as compared to CB AF ablation. However, on adjusted analysis to determine the predictors of atypical AFL, the predictive value of RF ablation was attenuated. Although we did not have further data related to index AF ablation to perform an adjusted predictor analysis, our study with a large sample size is important in suggesting that $\mathrm{CB}$ ablation of AF may be associated with a lower incidence of atypical AFL recurrence as compared to RF ablation. Similar to several other previously published studies(6,11-13), Our study suggests that roof dependent and perimitral atypical atrial flutters are common in pre ablated patients. Our results derived from a large sample size extend the support to the hypothesis that previous AF ablation predisposes to the development of perimitral and roof dependent flutter forms.

To the best of our knowledge, our study is the first with a relatively large sample size to evaluate the prognostic significance of recurrent atypical AFL after index AF ablation on the success of the repeat ablation. Ammar et al (14), in their small retrospective study, showed that recurrent atrial tachycardia after PsAF ablation is associated with a better success rate of repeat ablation procedure compared to recurrent persistent AF. In contrast, our study included both types of AF patients at the time of index ablation and comprised a greater proportion of PAF patients than PsAF patients. Additionally, we also demonstrated that patients undergoing repeat ablation for atypical AFL were older with dilated LA and higher LAVi compared to those presenting with recurrent AF; these factors are associated with poor ablation outcomes. Our results are interesting in demonstrating that despite the association with factors predictive of poor outcomes of the ablation procedure, patients undergoing repeat ablation for atypical AFL have a better success rate as compared to those for recurrent AF.

The findings of our study should be interpreted with attention to the associated limitations, including: 1) Limitations inherent to a single-center, retrospective, and observational study; 2) We did not have detailed data related to index $\mathrm{AF}$ ablation for all patients, precluding an adjusted analysis to determine predictive factors for atypical AFL recurrence; 3) LA diameter and LA volume were not available for all the included patients; 4) lack of continuous ECG monitoring during follow-up might have contributed to an underestimation of arrhythmia recurrence rate.

In conclusion, based on our experience, roof dependent, and perimitral flutter are the common forms of atypical AFL after index AF ablation. Patients developing atypical AFl after index AF ablation have dilated LA and higher LAVi and arrhythmia free survival rate of first repeat ablation is higher for patients presenting with recurrent atypical AFL as compared to recurrent AF.

\section{References}

1. Calkins H, Hindricks G, Cappato R et al. 2017 HRS/EHRA/ECAS/APHRS/SOLAECE expert consensus statement on catheter and surgical ablation of atrial fibrillation. Heart rhythm 2017;14:e275-e444.

2. Cappato R, Calkins H, Chen S-A et al. Updated worldwide survey on the methods, efficacy, and safety of catheter ablation for human atrial fibrillation. Circulation: Arrhythmia and Electrophysiology 2010;3:32-38.

3. Lang RM, Badano LP, Mor-Avi V et al. Recommendations for cardiac chamber quantification by echocardiography in adults: an update from the American Society of Echocardiography and the European Association 
of Cardiovascular Imaging. European Heart Journal-Cardiovascular Imaging 2015;16:233-271.

4. Wójcik M, Berkowitsch A, Zaltsberg S et al. Predictors of early and late left atrial tachycardia and left atrial flutter after catheter ablation of atrial fibrillation: Long-term follow-up. Cardiology journal 2015;22:557-566.

5. Ipek EG, Marine J, Yang E et al. Predictors and Incidence of Atrial Flutter After Catheter Ablation of Atrial Fibrillation. The American journal of cardiology 2019;124:1690-1696.

6. Chae S, Oral H, Good E et al. Atrial tachycardia after circumferential pulmonary vein ablation of atrial fibrillation: mechanistic insights, results of catheter ablation, and risk factors for recurrence. Journal of the American College of Cardiology 2007;50:1781-1787.

7. Sawhney N, Anousheh R, Chen W, Feld GK. Circumferential pulmonary vein ablation with additional linear ablation results in an increased incidence of left atrial flutter compared with segmental pulmonary vein isolation as an initial approach to ablation of paroxysmal atrial fibrillation. Circulation: Arrhythmia and Electrophysiology 2010;3:243-248.

8. Gerstenfeld EP, Callans DJ, Dixit S et al. Mechanisms of organized left atrial tachycardias occurring after pulmonary vein isolation. Circulation 2004;110:1351-7.

9. Juliá J, Chierchia G-B, de Asmundis C et al. Regular atrial tachycardias following pulmonary vein isolation for paroxysmal atrial fibrillation: a retrospective comparison between the cryoballoon and conventional focal tip radiofrequency techniques. Journal of Interventional Cardiac Electrophysiology 2015;42:161-169.

10. AKERSTRÖM F, Bastani H, Insulander P, Schwieler J, Arias MA, JENSEN-URSTAD M. Comparison of regular atrial tachycardia incidence after circumferential radiofrequency versus cryoballoon pulmonary vein isolation in real-life practice. Journal of cardiovascular electrophysiology 2014;25:948-952.

11. Siebermair J, Kochhauser S, Kupusovic J et al. Impact of previous left atrial ablation procedures on the mechanism of left atrial flutter: A single-centre experience. Journal of cardiovascular electrophysiology 2020 .

12. Peng H, Sun Z, Zhang H, Wu Y. Radiofrequency ablation of left atrial flutter mediated with double potentials in a seemingly normally structured heart. International journal of cardiology 2014;175:522-527.

13. Coffey JO, d'Avila A, Dukkipati S et al. Catheter ablation of scar-related atypical atrial flutter. Europace 2013;15:414-419.

14. Ammar S, Hessling G, Reents $\mathrm{T}$ et al. Arrhythmia type after persistent atrial fibrillation ablation predicts success of the repeat procedure. Circulation: Arrhythmia and Electrophysiology 2011;4:609-614.

\section{Hosted file}

Figure 1.pdf available at https://authorea.com/users/384507/articles/500126-ablationoutcomes-for-atypical-atrial-flutter-versus-recurrent-atrial-fibrillation-followingindex-pulmonary-vein-isolation

\section{Hosted file}

Figure 2.pdf available at https://authorea.com/users/384507/articles/500126-ablationoutcomes-for-atypical-atrial-flutter-versus-recurrent-atrial-fibrillation-followingindex-pulmonary-vein-isolation

\section{Hosted file}

Tables.pdf available at https://authorea.com/users/384507/articles/500126-ablation-outcomesfor-atypical-atrial-flutter-versus-recurrent-atrial-fibrillation-following-indexpulmonary-vein-isolation 